Portland State University

PDXScholar

8-1-1995

\title{
Ionization-excitation of helium by fast charged particles
}

\author{
L. Nagy \\ J. Wang \\ Jack C. Straton \\ Portland State University, straton@pdx.edu \\ James H. McGuire
}

Follow this and additional works at: https://pdxscholar.library.pdx.edu/phy_fac

Part of the Physics Commons

Let us know how access to this document benefits you.

\section{Citation Details}

Ionization-excitation of helium by fast, charged particles, L. Nagy, J. Wang, Jack C. Straton, and J. H. McGuire, Phys. Rev. A 52, R902 (1995).

This Article is brought to you for free and open access. It has been accepted for inclusion in Physics Faculty Publications and Presentations by an authorized administrator of PDXScholar. Please contact us if we can make this document more accessible: pdxscholar@pdx.edu. 


\title{
Ionization-excitation of helium by fast charged particles
}

\author{
L. Nagy, J. Wang, Jack C. Straton, ${ }^{*}$ and J.H. McGuire \\ Physics Department, Tulane University, New Orleans, Louisiana 70118-5698
}

(Received 13 April 1995)

\begin{abstract}
Probabilities and cross sections for ionization plus excitation in helium produced by fast heavy-particle impact have been evaluated. In these calculations, contributions from shake-off, time ordering, and independent interactions of the frozen-target electrons with the projectile are included. A comparison is made to recent experimental observations for the ratio of excitation-ionization to single-ionization total cross sections. A comparison is also made to calculations of excitation-ionization by fast electron impact.
\end{abstract}

PACS number(s): 34.50.Fa

Two-electron transitions in fast collisions with charged projectiles have been studied by various groups in the past several years both theoretically [1-7] and experimentally [8-12]. These studies yield information on the dynamics of the electron-electron interaction since two-electron transitions are dominated by the electron-electron interaction in very fast collisions. At moderately fast velocities, twoelectron transitions may be described [1] in terms of a coherent sum of first- and second-order terms in a Born expansion in the interaction strength (i.e., projectile charge) $Z$. The square of such a first- plus second-order amplitude yields a $Z^{3}$ contribution to physical observables such as transition probabilities and cross sections. Since the uncorrelated contribution to the second-order amplitude does not contribute the $Z^{3}$ term, such observable $Z^{3}$ effects may also be classified as dynamic correlation effects.

A two-electron transition that has been recently observed $[11,12]$ is ionization accompanied by excitation in helium when it is scattered from a charged particle. Calculations of this two-electron transition have been reported at low to moderate collision velocities by Rudge [13] and by Raeker et al. [14]. In this paper we report calculations of excitationionization in helium by impact of fast charged particles ranging from asymptotically large velocities where double to single cross section ratios tend to a constant to moderately high velocities where $Z^{3}$ terms occur.

For the calculation of the ionization-excitation cross section of the helium by charged particle impact we treat the projectile as a classical particle with a straight line trajectory, $\vec{R}(t)=\vec{b}+\vec{v} t$, and treat the electronic evolution separately [15]. The interaction of the projectile with the two electrons

$$
V(t)=V_{1}(t)+V_{2}(t)
$$

is considered as a perturbation. Instead of the exact unperturbed Hamiltonian of the two electrons, namely,

$$
H_{\text {exact }}^{0}=-\frac{1}{2}\left(\nabla_{1}^{2}+\nabla_{2}^{2}\right)-\frac{Z_{T}}{r_{1}}-\frac{Z_{T}}{r_{2}}+\frac{1}{r_{12}}
$$

\footnotetext{
*Present address: Department of Physics, Portland State University, Portland, OR 97207-0751.
}

the initial state is approximately described by a sum of oneelectron Hartree-Fock Hamiltonians. For the final state, because of the change in the screening, the Hamiltonian is taken,

$$
H^{0}=-\frac{1}{2} \nabla_{1}^{2}-\frac{Z_{T}}{r_{1}}+v_{e x}\left(\mathbf{r}_{1}\right)-\frac{1}{2} \nabla_{2}^{2}-\frac{Z_{T}}{r_{2}}
$$

Here electron 1 is the ejected one and is in the screening potential of electron 2, but the excited electron is not screened in the final state. Because in the initial state we have two electrons in the same $(1 s)$ state, the effect of electron symmetry may be ignored.

The first-order amplitude, with a single interaction with the projectile is

$$
\begin{aligned}
a^{(1)}= & -i\left\langle f_{2}^{e x} \mid i_{2}\right\rangle \int_{-\infty}^{+\infty} d t e^{i\left(\epsilon_{f_{1}}^{c}+\epsilon_{f_{2}}^{e x}-E_{0}\right) t}\left\langle f_{1}^{c}\left|V_{1}(t)\right| i_{1}\right\rangle \\
& -i\left\langle f_{1}^{c} \mid i_{1}\right\rangle \int_{-\infty}^{+\infty} d t e^{i\left(\epsilon_{f_{1}}^{c}+\epsilon_{f_{2}}^{e x}-E_{0}\right) t}\left\langle f_{2}^{e x}\left|V_{2}(t)\right| i_{2}\right\rangle,
\end{aligned}
$$

where $i$ stands for the initial state, and $f^{e x}$ and $f^{c}$ stand for the final excited and continuum states, respectively. $\epsilon_{f_{1}}^{c}$ and $\epsilon_{f_{2}}^{e x}$ are the final energies of the electrons, while $E_{0}$ is the ground-state energy of the helium. Because the initial and final states have different Hamiltonians, the overlap integrals in (4) can be nonzero. However, for the excitation of the $p$ states, only the overlap with the continuum is nonzero. This first-order term in the amplitude can be regarded as a shakeoff contribution.

In our second-order term the transition is caused by two consecutive projectile-electron interactions. We keep track of the time ordering: the energy transfer to the individual electron depends on the order of the interactions [16]. If the excitation occurs first, followed by an ionization, the energy transfer to the excited electron is $\Delta E^{e x}$, and the ionization potential is $\Delta E_{0}-\Delta E^{e x}$. If the ionization is first, then the ionization potential is $\Delta E^{i o n}$, and the excitation energy is $\Delta E_{0}-\Delta E^{i o n}$. Here $\Delta E_{0}=\epsilon_{f_{2}}^{e x}-E_{0}$ is the total energy transfer without the continuum state energy, and $\Delta E^{e x}$ and $\Delta E^{i o n}$ are the experimental single ionization and excitation energies. lows:

The second-order amplitude may now be written as fol- 


$$
\begin{aligned}
a^{(2)}= & -\int_{-\infty}^{+\infty} d t e^{i\left(\Delta E_{0}-\Delta E^{i o n}\right) t}\left\langle f_{2}^{e x}\left|V_{2}(t)\right| i_{2}^{\prime}\right\rangle \int_{-\infty}^{t} d t^{\prime} e^{i\left(\Delta E^{i o n}+\epsilon_{f_{1}}^{c}\right) t^{\prime}}\left\langle f_{1}^{c \prime}\left|V_{1}\left(i^{\prime}\right)\right| i_{1}\right\rangle \\
& -\int_{-\infty}^{+\infty} d t e^{i\left(\Delta E_{0}-\Delta E^{e x}+\epsilon_{f_{1}}^{c}\right) t}\left\langle f_{1}^{c}\left|V_{1}(t)\right| i_{1}^{\prime}\right\rangle \int_{-\infty}^{t} d t^{\prime} e^{i \Delta E^{e x} t^{\prime}}\left\langle f_{2}^{e x \prime}\left|V_{2}\left(t^{\prime}\right)\right| i_{2}\right\rangle .
\end{aligned}
$$

The wave functions in the matrix element for the first interaction in time are calculated in the initial-state Hamiltonian. In the matrix element for the second interaction the wave functions are calculated in the final-state Hamiltonian. This second-order contribution is often referred to as the two-step 2 (TS2) amplitude.

For a given final state the first-order amplitude is purely real or purely imaginary. The second-order amplitude, because of the time-ordering term, is complex. Interference occurs between first-order and second-order amplitudes, so we obtain different cross sections for positively and negatively charged projectiles.

The cross section can be calculated by integrating the square of the amplitude over the impact parameters

$$
\sigma=2 \int\left|a^{(1)}+a^{(2)}\right|^{2} d^{2} b
$$

where the factor 2 stands for the two possible equivalent final states: electron 1 in continuum and 2 excited or 2 in continuum and 1 excited.

Figure 1 shows our calculated ionization-excitation cross sections for the $2 p$ state of the helium by charged particle impact compared to the experimental data [17] and the theoretical results of Rudge [13]. Our cross sections for positive and negative projectiles differ only by a maximum of $8 \%$, although in the experimental data for protons and electrons there are differences up to a factor of 2 . The absolute values of our cross sections are about two times lower than the

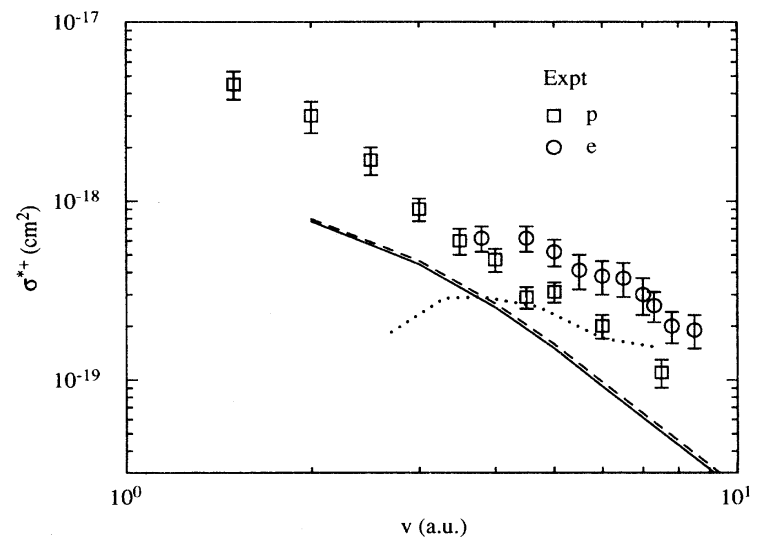

FIG. 1. Cross sections for the ionization and excitation of the $2 p$ state of helium by charged particle impact as a function of the projectile velocity. The solid line represents our calculation for protons; the dashed line for antiprotons. The theoretical results of Rudge [13] are represented by the dotted line. Experimental data are from Bruch [17]. experimental data for protons. At very high velocities we expect cross sections for $p^{+}, p^{-}, e^{-}$, and $e^{+}$to approach the same limit [1]. This limit is expected to be correctly described by a first-order term in $Z$, but complete to all orders in the electron-electron interaction. When a comparison with the theoretical calculations of Rudge is made, it is clear that for higher velocities (above 4 a.u.), when our semiclassical approximation is valid for electrons, our results are lower.

In our calculations the TS 2 contribution is dominant up to a velocity of 15 a.u. of the projectile. Above this velocity the shake-off contribution is higher. At 80 a.u. velocity the TS2 becomes only $3 \%$ of the shake-off cross section. Probably, the main reason for our results being below the experimental data is the neglect of the configuration interaction in the initial state.

We have made estimates for the importance of the configuration interaction (CI) contribution in the initial state, using a different code [18]. The second-order and the shakeoff amplitudes are not too sensitive to the inclusion of configurations other than the basic configuration, but by the inclusion of a configuration with a large overlap with the final $2 p$ state, the first term in Eq. (4) may contribute. This term can be interpreted as a shake-up amplitude. For the wave function described in Ref. [18], for high velocities, the shake-up term is $80 \%$ of the shake-off amplitude. However, the shake-up contribution to the amplitude is very sensitive to the CI wave function used, and is roughly proportional to the coefficient of the configuration exhibiting a large overlap with the final $2 p$ state. Different CI wave functions can give

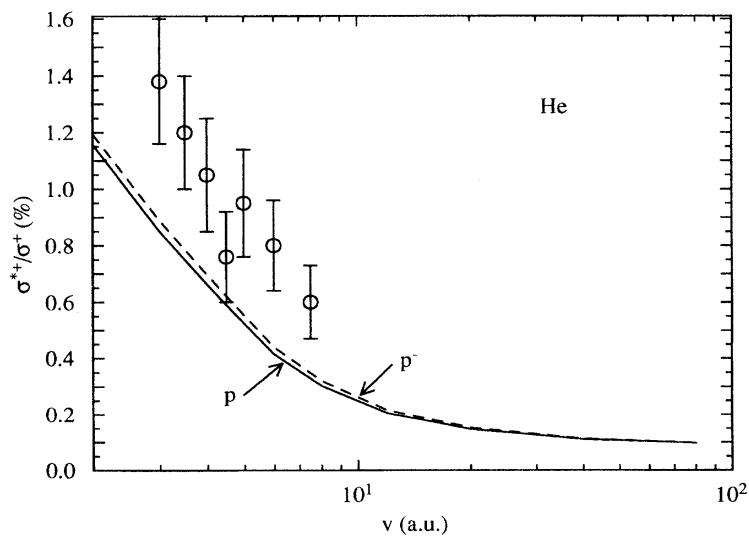

FIG. 2. Ratio of the ionization and excitation of the $2 p$ state cross section for helium to the single ionization cross section as a function of the projectile velocity. The solid line represents theoretical results for protons; the dashed line for antiprotons. Experimental data are for protons, and are taken from Bruch [17]. 
shake-up amplitudes differing by up to a factor of 2 .

In Fig. 2 is plotted the ratio of the ionization-excitation cross section to single ionization. In the high-velocity limit our ratio tends to a constant value of about $0.95 \%$. It is possible that the correct asymptotic value is as much as a factor of 2 larger than our value because of the neglect of some configuration interaction in initial and final states in our calculation.

In summary, we have calculated the ionization-excitation cross section of helium impacting on fast charged particles, taking into account both first-order and second-order processes, and keeping track of the time ordering. We obtain a small difference in cross sections for positively and negatively charged particle impact, but our calculated difference is lower than the factor of 2 difference observed. Our calculated sign is in agreement with observation. The absolute values of the cross sections are about two times lower than the experimental data. The reason for this discrepancy could be our neglect of some electron-electron effects in the initial and final states.

This work was supported by the Division of Chemical Sciences, Office of Basic Energy Sciences, Office of Energy Research, U.S. Department of Energy.
[1] J.H. McGuire, Adv. At. Mol. Opt. Phys. 29, 217 (1991).

[2] R.J. Tweed, Z. Phys. D 23, 309 (1992).

[3] J.F. Reading and A.L. Ford, J. Phys. B 20, 3747 (1987).

[4] M. Matsuzawa et al., in Abstracts of Contributed Papers of the XIXth International Conference on the Physics of Electronic and Atomic Collisions (Whistler, Canada), edited by B. Mitchell et al., AIP Conf. Proc. (AIP, New York, in press).

[5] F. Martin and A. Salin, J. Phys. B 28, 639 (1995).

[6] A.L. Gudunov and V.A. Schipakov, J. Phys. B 26, L811 (1993).

[7] L. Nagy and L. Végh, Phys. Rev. A 50, 3984 (1994).

[8] L.H. Andersen, P. Hvelplund, H. Knudsen, S.P. Moller, A.H. Sorensen, K. Elsner, K.G. Rensfeld, and E. Uggerhoj, Phys. Rev. A 36, 3612 (1987).

[9] J.P. Giese, M. Schulz, J.K. Swensen, H. Schoene, M. Benhennu, S.L. Varghese, C.R. Vane, P.F. Dittner, S.M. Shafroth, and S. Datz, Phys. Rev. A 42, 1231 (1990).
[10] A. Bordenave-Montesquieu et al., in Abstracts of Contributed Papers of the XIXth International Conference on the Physics of Electronic and Atomic Collisions (Whistler, Canada) (Ref. [4]).

[11] O. Pedersen and F. Folkmann, J. Phys. B 23, 441 (1990).

[12] S. Fülling, R. Bruch, E.A. Rauscher, P.A. Neil, E. Träbert, P.H. Heckmann, and J.H. McGuire, Phys. Rev. Lett. 68, 3152 (1992).

[13] M.R.H. Rudge, J. Phys. B 21, 1887 (1988).

[14] A. Raeker, K. Bartschat, and R.H.G. Reid, J. Phys. B 27, 3129 (1994).

[15] J.H. McGuire and O.L. Weaver, Phys. Rev. A 34, 2473 (1986).

[16] L. Végh, B. Sulik, and N. Stolterfoht, Phys. Rev. A 51, 3017 (1995).

[17] R. Bruch (private communication).

[18] Jack C. Straton, J.H. McGuire, and Zheng Chen, Phys. Rev. A 46, 5514 (1992). 